\title{
Long-term effect of early-life stress from earthquake exposure on working memory in adulthood
}

This article was published in the following Dove Press journal:

Neuropsychiatric Disease and Treatment

26 November 2015

Number of times this article has been viewed

\author{
$\mathrm{Na} \mathrm{Li}^{1-3, *}$ \\ Yumei Wang ${ }^{1-3, *}$ \\ Xiaochuan Zhaol-3 \\ Yuanyuan Gao ${ }^{1-3}$ \\ Mei Song ${ }^{1-3}$ \\ Lulu Yu ${ }^{1-3}$ \\ Lan Wang ${ }^{1-3}$ \\ Ning $\mathrm{Li}^{\mathrm{I}-3}$ \\ Qianqian Chen ${ }^{1-3}$ \\ Yunpeng $\mathrm{Li}^{1-3}$ \\ Jiajia Cai ${ }^{1-3}$ \\ Xueyi Wang ${ }^{1-3}$
}

'Department of Psychiatry, The First Hospital of Hebei Medical University, ${ }^{2}$ Mental Health Institute of Hebei Medical University, ${ }^{3}$ Brain Ageing and Cognitive Neuroscience Laboratory, Hebei, People's Republic of China

*These authors contributed equally to this work
Correspondence: Xueyi Wang Department of Psychiatry, The First Hospital of Hebei Medical University, Brain Ageing and Cognitive Neuroscience Laboratory, Mental Health Institute of Hebei Medical University, 89 Donggang Road, Yuhua District, Shijiazhuang 05003I, People's Republic of China $\mathrm{Tel}+8631185917113$

Fax +86 3II 859। 7II5

Email ydyywxy@।63.com
Objective: The present study aimed to investigate the long-term effect of 1976 Tangshan earthquake exposure in early life on performance of working memory in adulthood.

Methods: A total of 907 study subjects born and raised in Tangshan were enrolled in this study. They were divided into three groups according to the dates of birth: infant exposure (3-12 months, $n=274$ ), prenatal exposure ( $n=269$ ), and no exposure (born at least 1 year after the earthquake, $\mathrm{n}=364$ ). The prenatal group was further divided into first, second, and third trimester subgroups based on the timing of exposure during pregnancy. Hopkins Verbal Learning Test-Revised and Brief Visuospatial Memory Test-Revised (BVMT-R) were used to measure the performance of working memory. Unconditional logistic regression analysis was used to analyze the influential factors for impaired working memory.

Results: The Hopkins Verbal Learning Test-Revised scores did not show significant difference across the three groups. Compared with no exposure group, the BVMT-R scores were slightly lower in the prenatal exposure group and markedly decreased in the infant exposure group. When the BVMT-R scores were analyzed in three subgroups, the results showed that the subjects whose mothers were exposed to earthquake in the second and third trimesters of pregnancy had significantly lower BVMT-R scores compared with those in the first trimester. Education level and early-life earthquake exposure were identified as independent risk factors for reduced performance of visuospatial memory indicated by lower BVMT-R scores.

Conclusion: Infant exposure to earthquake-related stress impairs visuospatial memory in adulthood. Fetuses in the middle and late stages of development are more vulnerable to stress-induced damage that consequently results in impaired visuospatial memory. Education and early-life trauma can also influence the performance of working memory in adulthood.

Keywords: Tangshan earthquake, early life stress, working memory, chronic effect

\section{Introduction}

Working memory, evolving from the concept of short-term memory, refers to a memory system that processes and temporarily stores information necessary for language comprehension, visual image manipulation, reasoning, learning, information integration, and memory updating. Baddeley proposed a model of working memory, which was composed of three major components: central executive, phonological loop, and visuospatial sketchpad. ${ }^{1}$ In 2000, Baddeley added a new component to the model, the episodic buffer, and the revised model provides a better perspective on how the working memory functions to perform the complex cognitive tasks. ${ }^{2}$ Development of working memory starts in early childhood and advances gradually with age. Stress has been shown by numerous animal and human studies to influence the capacity of working memory. Previous studies have shown that the presence of severe stress in early life 
correlates with impaired spatial learning and development of neuropathologies in adulthood. ${ }^{3,4}$ A recent study in rats showed impaired spatial memory with prenatal exposure to noise stress. $^{5}$

On July 28, 1976, an earthquake with a magnitude of 7.8 hit Tangshan, People's Republic of China, and resulted in the deaths of 242,000 people and severe injuries of 164,000 people. Such catastrophic natural disaster places tremendous stress on infants and developing fetuses. Previous research in our laboratory showed that the cognitive functions were decreased in 18-year-old adults whose mothers experienced the Tangshan earthquake during pregnancy, and a more significant decline was observed in those who were at the gestational age of 4-6 or 7-9 months when the earthquake occurred. ${ }^{6}$ The objective of present study is to investigate the effect of prenatal or early-life exposure to earthquake on working memory in adulthood.

\section{Methods}

\section{Study subjects}

The subjects of present study were recruited from workers of Kailuan Mining Group in six coal reserve bases, two communities, and two related units. Based on the dates of birth, the study subjects were divided into three groups: infant exposure, prenatal exposure, and no exposure (Figure 1).

The inclusion criteria for the infant exposure group were born and raised in Tangshan; born between July 29, 1975 and April 28, 1976; exposed to the earthquake between 3 and 12 months of age.
The inclusion criteria for the prenatal exposure group were born and raised in Tangshan; born between July 29, 1976 and April 28, 1977; exposed to the earthquake in fetal period.

The inclusion criteria for no exposure group were born and raised in Tangshan; born between July 29, 1977 and April 28, 1978; born 1-1.9 years after the earthquake.

The following exclusion criteria were applied: the presence of infection, hypertension, epilepsy or seizures, diabetes, thyroid disease, the use of medication, drinking history, or other traumatic events in addition to earthquake during pregnancy; any form of mental illness in the past or present; unknown or missing information regarding the pregnancy during earthquake.

Informed consents were obtained from all study subjects before enrollment. The study was approved by the Ethics Committee of the First Affiliated Hospital of Hebei Medical University (No 2014005).

\section{Questionnaires and psychological evaluation}

The questionnaires and psychological evaluation were finished using a one-on-one method involving the study subjects and investigators who received unified training. The questionnaire included the demographic information, traumatic experiences during the earthquake, and related conditions during pregnancy. The information regarding the earthquake and pregnancy was obtained from the parents of study subjects or other insiders. The traumatic events were assessed by the Childhood Trauma

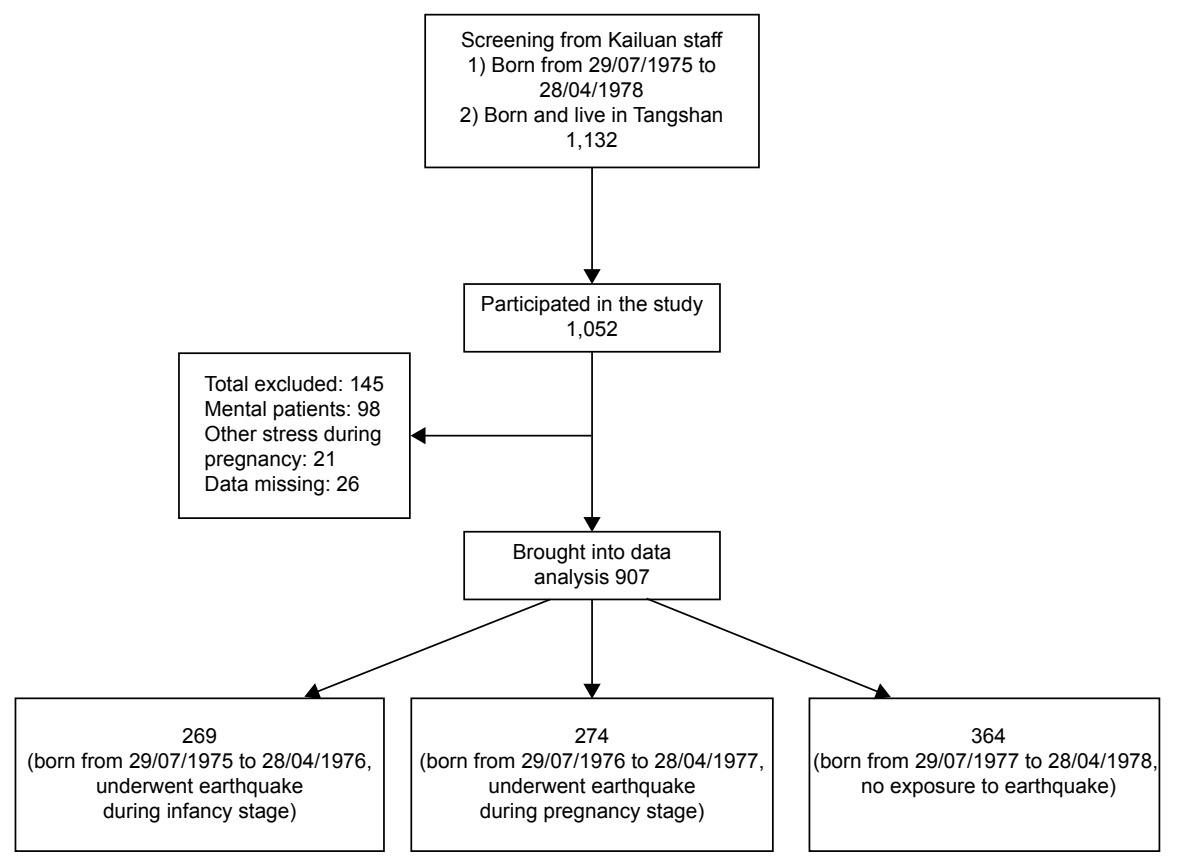

Figure I Inclusions and exclusions of subjects in the study. 
Questionnaire (CTQ) and Life Event Scale (LES). With the Diagnostic and Statistical Manual of Mental Disorders-4th edition (DSM-IV) being used as a diagnostic standard, the Structured Clinical Interview for DSM-IV was performed to screen and diagnose mental disorders in all study subjects.

\section{Assessment of working memory}

Hopkins Verbal Learning Test-Revised (HVLT-R) was used to assess the learning ability, immediate recall, and retention of verbal information. Brief Visuospatial Memory Test-Revised (BVMT-R) was used to assess the learning efficiency, immediate recall, and delayed recall in visuospatial memory. Each test was given to the same study subject three times and the results were recorded separately.

\section{Quality assurance}

All investigators in the study were psychiatrists and received unified training prior to investigation. Unified questionnaires and standardized guide language were used to ensure the consistency of findings and reduce bias. Consistency of $93 \%$ was obtained from all investigators. All questionnaires collected were reviewed by an independent group (quality assurance personnel) to verify the integrity, quality, and accuracy of the information.

\section{Statistical analysis}

SAS software version 9.2 was used for statistical analysis. Measurement data were expressed as the mean \pm standard deviation (mean $\pm \mathrm{SD}$ ). Comparison between groups was made by Wilcoxon rank-sum test, and comparison across multiple groups was made by Kruskal-Wallis test. Comparison of categorical data between groups was made by $\chi^{2}$ test. Unconditional logistic regression analysis was performed to analyze the risk factors.

\section{Results \\ Baseline characteristics and psychological evaluation of study subjects}

After screening the workers from Kailuan Mining Group, there were 1,132 individuals who met the inclusion criteria, and 1,052 agreed to participate in the study. After excluding 33 individuals who had incomplete or missing record and 98 with mental illness, a total of 921 individuals were enrolled in the study and signed informed consents. The completion rate was $81.4 \%(921 / 1,132)$. After completing the questionnaires and psychological evaluation, 14 individuals were further excluded due to missing information. Finally, 907 study subjects were included in our analysis.

The study subjects were divided into three groups based on their dates of birth: prenatal exposure $(n=274)$, infant exposure $(n=269)$, and no exposure $(n=364)$. The ages of subjects in the three groups showed significant difference $(P<0.001)$ (Table 1). The other baseline characteristics, including sex ratio and education level, did not differ across the three groups. The scores from LES, which measured the scale of stressful events each study subject experienced including loss of family members and houses during the earthquake, showed no significant differences between the two groups. When the study subjects were psychologically evaluated using the CTQ test, only a slightly higher score for physical neglect was observed in the prenatal exposure group compared with the other two groups.

\section{Comparison of working memory in different exposure groups}

HVLT-R and BVMT-R were performed in study subjects from the three groups to assess their verbal learning abilities and visuospatial memory, respectively. Test results were

Table I Baseline characteristics and psychological evaluation of study subjects

\begin{tabular}{|c|c|c|c|c|}
\hline & $\begin{array}{l}\text { No exposure } \\
(n=364)\end{array}$ & $\begin{array}{l}\text { Prenatal exposure } \\
(n=274)\end{array}$ & $\begin{array}{l}\text { Infant exposure } \\
(n=269)\end{array}$ & $P$-value* \\
\hline Age, mean \pm SD (years) & $37.02 \pm 1.36$ & $38.00 \pm 1.16 * *$ & $38.72 \pm 2.27$ & $<0.001$ \\
\hline Male, n (\%) & $316(87.05)$ & $245(89.42)$ & $236(88.06)$ & 0.660 \\
\hline High school diploma or higher, n (\%) & $330(90.66)$ & $235(85.77)$ & $232(86.25)$ & 0.108 \\
\hline LES scores, mean \pm SD & $22.5 I \pm 22.33$ & $20.87 \pm 22.83$ & $21.88 \pm 22.35$ & 0.433 \\
\hline \multicolumn{5}{|l|}{ CTQ scores, mean \pm SD } \\
\hline Emotional abuse & $5.80 \pm 1.96$ & $5.79 \pm 2.23$ & $5.80 \pm 1.82$ & 0.358 \\
\hline Emotional neglect & $8.88 \pm 3.86$ & $9.25 \pm 4.08$ & $8.72 \pm 3.46$ & 0.586 \\
\hline Sexual abuse & $5.47 \pm 2.09$ & $5.43 \pm 1.86$ & $5.45 \pm 1.47$ & 0.668 \\
\hline Physical neglect & $7.79 \pm 3.39$ & $8.12 \pm 2.78 * *$ & $7.54 \pm 2.57$ & 0.022 \\
\hline Physical abuse & $5.55 \pm 1.69$ & $5.51 \pm 1.93$ & $5.57 \pm 1.56$ & 0.097 \\
\hline Total CTQ scores & $33.73 \pm 12.41$ & $34.07 \pm 8.76$ & $33.12 \pm 7.84$ & 0.374 \\
\hline
\end{tabular}

Notes: *Represents comparison of three groups; **represents $P<0.05$ when compared with the no exposure and infant exposure groups.

Abbreviations: LES, Life Event Scale; CTQ, Childhood Trauma Questionnaire; SD, standard deviation. 
Table 2 Assessment of working memory in all study subjects

\begin{tabular}{cclll}
\hline & $\begin{array}{l}\text { No } \\
\text { exposure } \\
(\mathbf{n}=\mathbf{3 6 4})\end{array}$ & $\begin{array}{l}\text { Prenatal } \\
\text { exposure } \\
(\mathbf{n}=\mathbf{2 7 4})\end{array}$ & $\begin{array}{l}\text { Infant } \\
\text { exposure } \\
\mathbf{( n = 2 6 9 )}\end{array}$ & P-value* \\
\hline \multicolumn{2}{l}{ HVLT-R, mean \pm SD } & & & \\
I & $6.92 \pm 4.48$ & $6.81 \pm 2.16$ & $6.50 \pm 2.26$ & 0.172 \\
2 & $8.67 \pm 2.16$ & $8.69 \pm 2.21$ & $8.50 \pm 2.16$ & 0.408 \\
3 & $9.72 \pm 1.95$ & $9.53 \pm 2.11$ & $9.32 \pm 2.26$ & 0.122 \\
Total & $25.12 \pm 5.55$ & $25.03 \pm 5.70$ & $24.33 \pm 5.84$ & 0.175 \\
BVMT-R, mean \pm SD & & & \\
I & $6.94 \pm 3.19 * *$ & $6.40 \pm 3.28 * * *$ & $6.13 \pm 3.26$ & 0.003 \\
2 & $9.48 \pm 2.75 * *$ & $8.81 \pm 2.90^{* * *}$ & $8.79 \pm 3.12$ & 0.003 \\
3 & $10.58 \pm 2.19 * *$ & $10.17 \pm 2.45^{* * *}$ & $10.13 \pm 5.63$ & 0.006 \\
\multicolumn{1}{l}{ Total } & $26.64 \pm 7.88^{* *}$ & $25.35 \pm 7.75 * * *$ & $24.96 \pm 9.08$ & 0.006 \\
\hline
\end{tabular}

Notes: *Represents comparison of three groups; **represents $P<0.05$ when compared with the prenatal and infant exposure groups; $* * *$ represents $P>0.05$ when compared with the infant exposure group.

Abbreviations: BVMT-R, Brief Visuospatial Memory Test-Revised; HVLT-R, Hopkins Verbal Learning Test-Revised; SD, standard deviation.

analyzed and shown in Table 2. The scores from the 1st, 2nd, and 3rd HVLT-R and the total score did not show significant differences across three groups $(P>0.05)$, suggesting that the learning and language comprehension abilities in adulthood were not affected by prenatal or infant exposure to the earthquake. In contrast, those scores from BVMT-R in the no exposure group were markedly higher than those in the prenatal and infant exposure groups $(P<0.05$, Table 2$)$. There were no significant differences in all BVMT-R scores observed between the prenatal and infant exposure groups $(P>0.05)$. These results indicated that early-life exposure to the earthquake impaired the visuospatial memory in adulthood.

\section{Comparison of working memory within the prenatal exposure group}

To further evaluate the impact of earthquake on working memory in adulthood when the subjects were exposed prenatally, the prenatal exposure group was divided into three subgroups, first trimester, second trimester, and third trimester, depending on their gestational age when the earthquake occurred. The test scores from HVLT-R and BVMT-R were analyzed for three subgroups, and the results are shown in Table 3. The HVLT-R scores did not show significant differences, whereas the BVMT-R scores differed slightly across three subgroups. Further analysis showed that the second trimester subgroup had markedly decreased 2nd and total BVMT-R scores, and the third trimester subgroup had significantly lower total score and scores from the 2 nd and 3rd tests compared with those from the first trimester subgroup. These results suggested that the earthquake exposure had varying influences on fetuses in different developmental
Table 3 Assessment of working memory within the prenatal exposure group

\begin{tabular}{|c|c|c|c|c|}
\hline & $\begin{array}{l}\text { First } \\
\text { trimester }\end{array}$ & $\begin{array}{l}\text { Second } \\
\text { trimester }\end{array}$ & $\begin{array}{l}\text { Third } \\
\text { trimester }\end{array}$ & $P$-value* \\
\hline $\bar{N}$ & 88 & 91 & 95 & \\
\hline \multicolumn{5}{|c|}{ HVLT-R, mean $\pm S D$} \\
\hline 1 & $7.00 \pm 2.27$ & $6.63 \pm 2.13$ & $6.80 \pm 2.11$ & 0.262 \\
\hline 2 & $8.87 \pm 2.11$ & $8.68 \pm 2.34$ & $8.53 \pm 2.17$ & 0.561 \\
\hline 3 & $9.63 \pm 1.92$ & $9.63 \pm 2.09$ & $9.36 \pm 2.30$ & 0.716 \\
\hline Total & $25.5 I \pm 5.7 I$ & $24.93 \pm 5.59$ & $24.68 \pm 5.83$ & 0.604 \\
\hline \multicolumn{5}{|c|}{ BVMT-R, mean $\pm S D$} \\
\hline 1 & $7.04 \pm 3.14$ & $6.05 \pm 3.40$ & $6.13 \pm 3.24$ & 0.069 \\
\hline 2 & $9.52 \pm 2.46$ & $8.4 I \pm 3.0 \mathrm{I} * *$ & $8.55 \pm 3.07 * *$ & 0.035 \\
\hline 3 & $10.68 \pm 1.99$ & $10.05 \pm 2.54$ & $9.80 \pm 2.68 * *$ & 0.038 \\
\hline Total & $27.25 \pm 6.69$ & $24.52 \pm 8.03 * *$ & $24.39 \pm 8.13^{* *}$ & 0.038 \\
\hline
\end{tabular}

Notes: *Represents comparison of three groups; **represents $P<0.05$ when compared with the first trimester subgroup.

Abbreviations: BVMT-R, Brief Visuospatial Memory Test-Revised; HVLT-R, Hopkins Verbal Learning Test-Revised; SD, standard deviation.

stages, which resulted in different outcomes in visuospatial memory.

\section{Comparison of working memory within the infant exposure group}

Similarly, to evaluate the influence of earthquake on working memory in adulthood when the subjects were exposed at different ages during infancy, the infant exposure group was divided into three subgroups by age: $<6$ months, $6-9$ months, and $\geq 9$ months. The test scores from HVLT-R and BVMT-R were analyzed for three subgroups. There were no significant differences observed in either HVLT-R or BVMT-R scores across three subgroups (Table 4), suggesting that the earthquake exposure had undistinguished impact on infants of different ages.

Table 4 Assessment of working memory within the infant exposure group

\begin{tabular}{|c|c|c|c|c|}
\hline & $<6$ months & 6-9 months & $\geq 9$ months & $P$-value* \\
\hline $\mathrm{N}$ & 105 & 88 & 76 & \\
\hline \multicolumn{5}{|c|}{ HVLT-R, mean $\pm S D$} \\
\hline I & $6.20 \pm 2.34$ & $6.56 \pm 2.20$ & $6.68 \pm 2.25$ & 0.254 \\
\hline 2 & $8.21 \pm 2.16$ & $8.50 \pm 2.33$ & $8.70 \pm 2.00$ & 0.216 \\
\hline 3 & $9.09 \pm 2.52$ & $9.26 \pm 2.20$ & $9.52 \pm 2.12$ & 0.506 \\
\hline Total & $23.54 \pm 5.90$ & $24.34 \pm 6.02$ & $24.90 \pm 5.62$ & 0.210 \\
\hline \multicolumn{5}{|c|}{ BVMT-R, mean \pm SD } \\
\hline I & $6.45 \pm 3.41$ & $5.98 \pm 2.98$ & $6.02 \pm 3.40$ & 0.602 \\
\hline 2 & $8.89 \pm 3.20$ & $8.83 \pm 2.88$ & $8.68 \pm 3.28$ & 0.889 \\
\hline 3 & $11.20 \pm 9.59$ & $9.75 \pm 2.59$ & $9.68 \pm 2.92$ & 0.096 \\
\hline Total & $26.22 \pm 10.81$ & $24.55 \pm 7.63$ & $24.38 \pm 8.83$ & 0.415 \\
\hline
\end{tabular}

Note: *Represents comparison of three groups.

Abbreviations: BVMT-R, Brief Visuospatial Memory Test-Revised; HVLT-R, Hopkins Verbal Learning Test-Revised; SD, standard deviation. 
Table 5 Risk factor analysis of impaired visuospatial memory (BVMT-R total scores $<27$ )

\begin{tabular}{|c|c|c|c|c|}
\hline \multirow[t]{2}{*}{ Variable } & \multirow[t]{2}{*}{ OR } & \multicolumn{2}{|c|}{$95 \% \mathrm{Cl}$ of $\mathrm{OR}$} & \multirow[t]{2}{*}{$P$-value } \\
\hline & & Lower & Upper & \\
\hline Age & 1.044 & 0.958 & 1.137 & 0.331 \\
\hline Emotional abuse & 1.128 & 1.019 & 1.248 & 0.021 \\
\hline Emotional neglect & 0.963 & 0.922 & 1.006 & 0.094 \\
\hline Physical abuse & 1.011 & 0.900 & 1.137 & 0.850 \\
\hline Physical neglect & 1.121 & 1.054 & 1.192 & $<0.001$ \\
\hline Sexual abuse & 0.869 & 0.775 & 0.976 & 0.018 \\
\hline \multicolumn{5}{|l|}{ Sex } \\
\hline Male vs female & I.I77 & 0.761 & 1.819 & 0.464 \\
\hline LES scores & 0.998 & 0.992 & 1.004 & 0.510 \\
\hline \multicolumn{5}{|l|}{ Education level } \\
\hline $\begin{array}{l}\text { Less than a high school diploma } \\
\text { vs high school diploma or higher }\end{array}$ & 2.289 & 1.435 & 3.650 & $<0.001$ \\
\hline \multicolumn{5}{|l|}{ Earthquake exposure } \\
\hline $\begin{array}{l}\text { Prenatal and infant exposure vs } \\
\text { no exposure }\end{array}$ & 1.437 & 1.061 & 1.947 & 0.019 \\
\hline
\end{tabular}

Abbreviations: BVMT-R, Brief Visuospatial Memory Test-Revised; $\mathrm{Cl}$, confidence interval; LES, Life Event Scale; OR, odds ratio.

\section{Analysis of influential factors for visuospatial memory}

The total scores of BVMT-R from all study subjects were not normally distributed. Therefore, we used the median of all BVMT-R total scores (median $=27$ ) to divide them into two categories: $<27$ and $\geq 27$. The BVMT-R total scores were used as dependent variables, and after adjusting for age, sex, education level, LES, and CTQ scores, unconditional logistic regression analysis was performed to analyze the risk factors for impaired visuospatial memory in adulthood. In addition, because the prenatal and infant exposure groups did not have significant difference in visuospatial memory (Table 2), data from the two groups were combined and compared with the no exposure group. The results for risk analysis of impaired visuospatial memory in $<27$ group are shown in Table 5. Education level and earthquake exposure were shown as major risk factors for visuospatial memory (odds ratio $=2.289,95 \%$ confidence interval $=1.435-3.650$ and odds ratio $=1.437,95 \%$ confidence interval $=1.061-1.947$, respectively). Furthermore, emotional abuse, physical neglect, and sex were all associated with reduced performance of visuospatial memory in varying degrees.

\section{Discussion}

The present study investigated the impact of prenatal and early-life exposure to large earthquake on working memory in adulthood. The performance of working memory was assessed by HVLT-R and BVMT-R to evaluate the verbal learning and visuospatial memory, respectively. Among the study subjects in the prenatal and infant exposure groups, more than half of them had collapsed houses and lost relatives during the 1976 Tangshan earthquake, which caused a lot of stress on the pregnant women and nursing mothers. In addition, the harsh living conditions and inadequate water and housing supplies after the earthquake had direct or indirect impacts on the developing fetuses and infants. While the study subjects in the no exposure group, who were born at least 1 year after the earthquake, did not experience the earthquake-related stress prenatally and during infancy, as the living conditions had been gradually and greatly improved since the earthquake. By comparing the performance of working memory in adulthood across the three groups of study subjects, we found out that the subjects who experienced earthquake during infancy showed significantly lower BVMT-R scores than those with no exposure to earthquake, suggesting that the earthquake-related stress could reduce the performance of visuospatial memory in adulthood. Although the prenatal exposure group only showed slightly decreased BVMT-R scores compared with the no exposure group, after analyzing the results according to the gestational ages of the fetuses when the earthquake hit, the subjects whose mothers were in the second or third trimester of pregnancy were more severely affected by the earthquake.

In our previous study on the impact of prenatal exposure to earthquake on the cognitive functions in young adults, the earthquake-related stress showed a significantly negative effect on the cognitive functions of 18-year-old study subjects whose mothers were at the second or third trimester of pregnancy when the earthquake occurred compared with those who did not have prenatal exposure to earthquake. ${ }^{6}$ It has been shown that the prefrontal cortex, parietal cortex, occipital cortex, and cerebellum all participate in the visuospatial memory. ${ }^{7-10}$ Hippocampus has been implicated by many investigations to function as a cognitive map to recognize and retain spatial information. ${ }^{11,12}$ Recent studies have further demonstrated the important role hippocampus plays in processing spatial and nonspatial information for working memory. ${ }^{13}$ The second trimester of pregnancy is the fastest growth period of fetal hippocampus, which makes it more vulnerable to stress-related damage. In an animal study, female rats in the mid-to-late or late period of pregnancy were subjected to restraint stress, and the offspring from the mid-to-late pregnancy group showed more severely impaired neurodevelopment and hippocampal microstructure. ${ }^{14}$ Mechanistic studies in rodent models have suggested that the prenatal stress has a profound negative impact on spatial learning and memory in animals when 
they reach adulthood, which may be associated with deficits of $N$-methyl-D-aspartate receptor-mediated hippocampal synaptic plasticity. ${ }^{15,16}$ In our study, the performance of visuospatial memory in adulthood reduced more significantly in subjects whose mothers were in the middle or late stages of pregnancy when exposed to the earthquake-related stress. Our results were consistent with previous findings and indicated a critical role for the timing of stress exposure in development of visuospatial working memory.

Infancy refers to the period of early childhood (0-1 year), marked by accelerated physical and mental development. Catastrophic natural disaster such as the 1976 Tangshan earthquake caused serious physical and emotional damages to the infants in addition to nutritional deficiency, which has pronounced long-term effects on the cognitive functions and working memory in adulthood. ${ }^{6,17}$ During infancy, the brain and nervous system undergo rapid development. The function of hippocampus is essential for processing of spatial information, and child's early development is regulated by the hypothalamic-pituitary-adrenal axis and is thus vulnerable to stress-related damage. ${ }^{18}$ Animal studies have demonstrated that early-life exposure to stress results in morphological changes in brain regions related to cognitive functions in adults, including reduced hippocampal volume, decreased sizes of pyramidal and granular cells, decreased thickness of neocortex, and reduced density of neurons in retrosplenial and prefrontal cortex. ${ }^{19}$ These two regions are the neural basis for memory and learning. Meanwhile, the role of growth hormone in the development and performance of working memory has been well-documented. Acute exposure to growth hormone greatly improves the spatial working memory in rats. ${ }^{20}$ Majority of the growth hormone is produced in the pituitary gland and secreted into the circulating blood, and the rest is produced in the hippocampus and other part of the brain. ${ }^{21}$ Damages to the hippocampus and the hypothalamic-pituitary-adrenal axis caused by traumatic events could affect the synthesis of growth hormone and thus impair the normal development of cognitive functions. Similarly, thyroid function and production of thyroid hormones are profoundly influenced by stress and stress-related damages. Dysregulated thyroid hormones have been linked to impaired cognitive functions in the elderly. ${ }^{22}$ Therefore, we could not rule out the possibility that the variations in thyroid function caused by earthquake-related stress in the subjects who experienced earthquake during infancy lead to reduced performance of visuospatial memory in adulthood. In addition, the forebrain corticotropin-releasing hormone receptor 1 plays an important role in stress response in early life, and the impairment of working memory in adulthood has been proposed to result from the interaction of early-life stress and corticotropin-releasing hormone receptor 1 susceptible genotype. ${ }^{23,24}$ As for now, studies on the underlying biological mechanism of early stress exposure induced damage to adult visuospatial memory remain inconclusive.

The risk analysis in our study implicated education and prenatal or infant exposure to earthquake as major influencing factors, and childhood emotional abuse or physical neglect as minor risk factors for impaired visuospatial memory in adulthood. Ardila and Moreno proposed that education level could not only affect the verbal expression and concept formation, but also the visuospatial construction, visuoperception, memory, and other cognitive functions. ${ }^{25}$ The study from Gould et al showed a strong correlation between the experience of childhood emotional neglect or abuse and reduction in adult cognitive functions in patients with depression. ${ }^{26}$ Our results provide additional evidence showing the negative long-term effect of early-life trauma on neurodevelopment and working memory.

The present study investigated the impact of prenatal or early childhood trauma on working memory in adulthood. It is worth noting that many other factors may confound the analysis, such as genetic background, life events in adulthood, lifestyle, diet, alcohol, and drug abuse, but it is difficult to perform quantitative analysis on these confounding factors. In addition, we only assessed the working memory in study subjects with prenatal or infant exposure to earthquake, but no imaging or biological studies were performed to further explore the underlying mechanism. Investigation with a larger sample size and combined biological examination should be conducted to elucidate the role and working principle of early-life trauma on development of working memory.

\section{Acknowledgments}

This work was supported by the National Nature Science Foundation of China (81271489) and Nature Science Foundation General Programs of Hebei Province (2014206280).

\section{Disclosure}

The authors report no conflicts of interest in this work.

\section{References}

1. Baddeley A. Working memory. Science. 1992;255(5044):556-559.

2. Baddeley A. The episodic buffer: a new component of working memory? Trends Cogn Sci. 2000;4(11):417-423.

3. Oomen CA, Soeters H, Audureau N, et al. Severe early life stress hampers spatial learning and neurogenesis, but improves hippocampal synaptic plasticity and emotional learning under high-stress conditions in adulthood. J Neurosci. 2010;30(19):6635-6645. 
4. Oitzl MS, Workel JO, Fluttert M, Frosch F, De Kloet ER. Maternal deprivation affects behaviour from youth to senescence: amplification of individual differences in spatial learning and memory in senescent Brown Norway rats. Eur J Neurosci. 2000;12(10):3771-3780.

5. Barzegar M, Sajjadi FS, Talaei SA, Hamidi G, Salami M. Prenatal exposure to noise stress: anxiety, impaired spatial memory, and deteriorated hippocampal plasticity in postnatal life. Hippocampus. 2015; 25(2):187-196.

6. Wang X, Zhang B, Zhang B, et al. Long-term effect of earthquake on fetal cognitive function. Chin Mental Health J. 2001;15(1):42-43.

7. Jones KT, Berryhill ME. Parietal contributions to visual working memory depend on task difficulty. Front Psychiatry. 2012;3:81.

8. Hanson JL, Chung MK, Avants BB, et al. Structural variations in prefrontal cortex mediate the relationship between early childhood stress and spatial working memory. J Neurosci. 2012;32(23):7917-7925.

9. Makovski T, Lavidor M. Stimulating occipital cortex enhances visual working memory consolidation. Behav Brain Res. 2014;275:84-87.

10. Baier B, Muller NG, Dieterich M. What part of the cerebellum contributes to a visuospatial working memory task? Ann Neurol. 2014;76(5): 754-757.

11. Nadel L, MacDonald L. Hippocampus: cognitive map or working memory? Behav Neural Biol. 1980;29(3):405-409.

12. Manns JR, Eichenbaum H. A cognitive map for object memory in the hippocampus. Learn Mem. 2009;16(10):616-624.

13. Banta Lavenex PA, Colombo F, Ribordy Lambert F, Lavenex P. The human hippocampus beyond the cognitive map: evidence from a densely amnesic patient. Front Hum Neurosci. 2014;8:711.

14. Xu J, Yang B, Yan C, et al. Effects of duration and timing of prenatal stress on hippocampal myelination and synaptophysin expression. Brain Res. 2013;1527:57-66.

15. Xu C, Li S, Jiang H, Shao K, Zhang R. Effect of alcohol exposure during pregnancy on learning and memory and expression of N-methyl-Daspartate receptor 2B subunit in the hippocampus of infant rats. Chin J Psychiatry. 2011;44(4):235-239.
16. Son GH, Geum D, Chung S, et al. Maternal stress produces learning deficits associated with impairment of NMDA receptor-mediated synaptic plasticity. J Neurosci. 2006;26(12):3309-3318.

17. Prado EL, Dewey KG. Nutrition and brain development in early life. Nutr Rev. 2014;72(4):267-284.

18. Kim JJ, Diamond DM. The stressed hippocampus, synaptic plasticity and lost memories. Nat Rev Neurosci. 2002;3(6):453-462.

19. Aksic M, Radonjic NV, Aleksic D, et al. Long-term effects of the maternal deprivation on the volume and number of neurons in the rat neocortex and hippocampus. Acta Neurobiol Exp (Wars). 2013;73(3): 394-403.

20. Ramis M, Sarubbo F, Sola J, et al. Cognitive improvement by acute growth hormone is mediated by NMDA and AMPA receptors and MEK pathway. Prog Neuropsychopharmacol Biol Psychiatry. 2013; 45:11-20.

21. Sun LY, Al-Regaiey K, Masternak MM, Wang J, Bartke A. Local expression of GH and IGF-1 in the hippocampus of GH-deficient longlived mice. Neurobiol Aging. 2005;26(6):929-937.

22. Begin ME, Langlois MF, Lorrain D, Cunnane SC. Thyroid function and cognition during aging. Curr Gerontol Geriatr Res. 2008:474868.

23. Ivy AS, Rex CS, Chen Y, et al. Hippocampal dysfunction and cognitive impairments provoked by chronic early-life stress involve excessive activation of CRH receptors. J Neurosci. 2010;30(39):13005-13015.

24. Fuge P, Aust S, Fan Y, et al. Interaction of early life stress and corticotropin-releasing hormone receptor gene: effects on working memory. Biol Psychiatry. 2014;76(11):888-894.

25. Ardila A, Moreno S. Neuropsychological test performance in Aruaco Indians: an exploratory study. J Int Neuropsychol Soc. 2001;7(4): 510-515.

26. Gould F, Clarke J, Heim C, Harvey PD, Majer M, Nemeroff CB. The effects of child abuse and neglect on cognitive functioning in adulthood. J Psychiatr Res. 2012;46(4):500-506.
Neuropsychiatric Disease and Treatment

\section{Publish your work in this journal}

Neuropsychiatric Disease and Treatment is an international, peerreviewed journal of clinical therapeutics and pharmacology focusing on concise rapid reporting of clinical or pre-clinical studies on a range of neuropsychiatric and neurological disorders. This journal is indexed on PubMed Central, the 'PsycINFO' database and CAS,

\section{Dovepress}

and is the official journal of The International Neuropsychiatric Association (INA). The manuscript management system is completely online and includes a very quick and fair peer-review system, which is all easy to use. Visit http://www.dovepress.com/testimonials.php to read real quotes from published authors. 